\title{
A resiliência em idosos e sua relação com variáveis sociodemográficas e funções cognitivas ${ }^{1}$
}

\author{
Resilience in the elderly and its relationship with \\ socio-demographic variables and cognitive functions
}

\author{
Tatiane Favarin Rech FORTES ${ }^{2}$ \\ Mirna Wetters PORTUGUEZ ${ }^{2}$ \\ Irani Iracema de Lima ARGIMON $^{3}$
}

\begin{abstract}
Resumo
Este artigo objetivou estudar a resiliência do idoso e sua relação com as variáveis sociodemográficas e funções cognitivas. Para tanto, relacionou os escores obtidos por meio da Escala de Resiliência com os escores de outros dois instrumentos de avaliação: Percepção Subjetiva de Queixas de Memória e Miniexame do Estado Mental. A amostra foi constituída por 86 idosos: 67 do sexo feminino e 19 do sexo masculino, com idade variando entre 60 e 90 anos. Os resultados revelaram que não houve relação entre resiliência e as variáveis demográficas. Demonstraram, entretanto, que um número mais elevado de queixas subjetivas de memória correspondia a baixos índices de resiliência. Também revelou haver uma correlação direta e significativa entre a Escala de Resiliência e o Miniexame do Estado Mental. Deste modo, conclui-se que quanto mais altos os escores de resiliência, maiores os escores no desempenho cognitivo entre os idosos.
\end{abstract}

Unitermos: Dados demográficos. Idosos. Resiliência. Psicologia.

\begin{abstract}
The aim of this article is to study the relationship between older people's resilience and some socio-demographic variables and cognitive functions. In order to do so, the scores obtained from the Resilience Scale were compared to average scores from two other investigation tools: Subjective Perception of Memory Complaints and the Mini-Mental State Examination. The research sample was composed of 86 old people, of which 67 were women, and 19 were men, whose ages varied from 60 to 90 . The results showed that there is no relationship between resilience and socio-demographic variables. On the other hand, they did show that higher numbers of memory subjective complaints directly correspond to lower indices of resilience. The survey also revealed that there is a direct and meaningful correlation between the Resilience Scale and Mini-Mental State Examination, so it is possible to conclude that the higher the resilience scores, the greater are old people's achievements in cognitive performance scoring.
\end{abstract}

Uniterms: Demographic data. Aged. Resilience. Psychological.

$\boldsymbol{\nabla} \mathbf{\nabla} \boldsymbol{\nabla}$

1 Artigo elaborado a partir da dissertação de T.F.R. FORTES, intitulada "A resiliência em idosos e sua relação com as variáveis sócio-demográficas: gênero, idade, escolaridade e renda". Pontifícia Universidade Católica do Rio Grande do Sul, 2007.

2 Pontifícia Universidade Católica do Rio Grande do Sul, Faculdade de Medicina, Programa de Pós-Graduação em Medicina e Ciências da Saúde. Av. Ipiranga, 6681, 90619-900, Porto Alegre, RS, Brasil. Correspondência para/Correspondence to: T.F.R. FORTES. E-mail: <tatifrech@pop.com.br>.

3 Pontifícia Universidade Católica do Rio Grande do Sul, Programa de Pós-Graduação Psicologia Clínica. Porto Alegre, RS, Brasil. 
As projeções estatísticas entre 1950 e 2025 são de que a população de idosos no Brasil crescerá 16 vezes contra 5 vezes a população total. Isso colocará o País como a sexta população de idosos do mundo, com mais de 32 milhões de pessoas de 60 anos ou mais (Organización Mundial de La Salud - OMS, 1984). As projeções estatísticas demonstram que a proporção de idosos no país passará de 7,3\%, em 1991, para quase 15\%, em 2025, assemelhando à proporção de idosos da maioria dos países europeus (Costa, Guerra, Barreto \& Guimarães, 2000).

A população idosa aumenta sensivelmente, apresentando, assim, um desafio à competência dos profissionais quanto ao entendimento de seus problemas específicos (Costa et al., 2000). A chamada terceira idade apresenta problemas peculiares; seu atendimento requer um aumento de cuidados já previstos e dispensados a outros grupos etários, assim como estratégias a serem utilizadas para avaliar as questões características destafase.

A velhice é frequentemente descrita como um período caracterizado por uma diminuição das reservas, em virtude das múltiplas perdas que ocorrem simultânea ou sucessivamente no decorrer de um curto período de tempo. Eventos negativos não normativos e graduados por idade, como morte do cônjuge e de amigos, declínio da saúde e da funcionalidade física, perda de status social e prestígio e, às vezes, insegurança econômica, além da proximidade crescente da morte, tornam-se predominantes na velhice avançada. De fato, alguns eventos raros ou não normativos nas fases anteriores (por exemplo, doenças) tornam-se relativamente normativos na velhice.

Consideradas em conjunto, essas perspectivas de risco e desafios sugerem que é necessário um aumento na capacidade de reservas e da resiliência na velhice para que o funcionamento adaptativo possa se manter (Schaie \& Hofer, 2001).

A resiliência é comumente definida como a capacidade do indivíduo ou da família de enfrentar as adversidades, ser transformado por elas e conseguir superá-las. Assim, por resiliência entende-se o conjunto de processos sociais e intrapsíquicos que possibilitam o desenvolvimento saudável da pessoa, mesmo esta vivenciando experiências desfavoráveis (Pinheiro, 2004).
De acordo com Melillo, Suárez-Ojeda e Rodríguez (2004), o conceito de resiliência nasceu e se desenvolveu no Hemisfério Norte (Michael Rutter, na Inglaterra, e Emmy Werner, nos Estados Unidos), logo se estendendo por toda a Europa, especialmente na França, Países Baixos, Alemanha e Espanha. Mais tarde chegou à América Latina, onde foram estabelecidos importantes grupos de investigação e numerosos projetos específicos. Segundo esses autores existiriam três bases teóricas de estudos a respeito: a norte-americana, essencialmente pragmática e centrada no individual; a europeia, com maiores enfoques psicanalíticos e uma perspectiva ética; e a latino-americana, de enfoque comunitário, voltada para o social como resposta lógica aos problemas vivenciados naquela sociedade, tais como violência, marginalidade, vulnerabilidade etc.

A resiliência possui várias definições, revelando que o conceito encontra-se em fase de construção e debate. Originariamente, o termo resiliência vem da física e refere-se à "propriedade pela qual a energia armazenada em um corpo deformado é devolvida quando cessa a tensão causadora da deformação elástica" (Ferreira, 1975, p.1223). A metáfora mais evocada é a de um elástico que, após uma tensão inicial, volta ao mesmo estado. Porém, concernente ao ser humano, a resiliência não significa um retorno a um estado anterior, mas a superação (ou adaptação, como querem alguns autores) diante de uma dificuldade considerada um risco e a possibilidade de construção de novos caminhos de vida e de um processo de subjetivação a partir do enfrentamento de situações estressantes e/ou traumáticas. Desta forma, seria uma capacidade (singular e também socialmente adquirida) de sair-se bem frente a fatores potencialmente estressores (Lindström, 2001; Vanistendael, 1999).

É relevante ressaltar que o tema resiliência, no Brasil, ainda é recente. Em uma revisão de literatura realizada por Souza e Cerveny (2006), constatou-se que os primeiros trabalhos no país relacionados à resiliência surgiram entre 1996 e 1998, com estudos sobre crianças expostas a situação de risco, fatores de proteção e vulnerabilidade psicossocial (Hutz, 1996; Hutz, Koller \& Bandeira, 1996), redes de apoio social e afetivo de criança em situação de risco (Hoppe, 1998) e na área ocupacional, 
associando a resiliência ao perfil do executivo (Giuliani, 1997). Na verdade, as pesquisas sobre resiliência como tema central ou associada a outros aspectos tiveram seu desenvolvimento maior nos últimos cinco anos (Souza \& Cerveny, 2006).

Diante de perdas, o self apresenta resiliência ou capacidade de reserva, isto é, o potencial de manutenção e recuperação dos níveis de adaptação normal e a presença de recursos latentes podem ser ativados frente aos desafios e exigências (Staudinger, Marsiske \& Baltes, 1995). É proposto que os idosos, mesmo sob condições de limitações e incapacidades funcionais, mantenham um senso positivo de bem-estar (Smith, Bahelt, Maier \& Jopp 2002). Perdas na funcionalidade não são, necessariamente, um impedimento para a continuidade do funcionamento cognitivo e emocional; como qualquer ser humano, o idoso consegue ativar mecanismos compensatórios para lidar com essas perdas (Neri, 2001).

Se é verdade que o mundo está envelhecendo, com o processo de envelhecimento torna-se necessário o aumento na capacidade de resiliência na velhice para manter o comportamento adaptativo, pois é maior a probabilidade de ocorrerem na velhice eventos desagradáveis relacionados à saúde física e ao bem-estar e relacionados à vida de entes queridos. Isto não significa que os fatores protetores não funcionem na velhice; entretanto, em uma velhice avançada, as chances de experienciar vários eventos ao mesmo tempo são bem maiores do que quando se é jovem (Pinheiro, 2004).

Assim, estudar a resiliência da terceira idade é relevante, não apenas porque profissionais dedicados à gerontologia têm explorado pouco este tema, mas, principalmente, porque é um aporte inovador que abre portas para uma área de grande futuro na investigação e na aplicação prática de recursos que melhorem a qualidade de vida dos idosos.

Portanto, este trabalho teve como objetivo estudar a resiliência nos idosos e sua relação com as variáveis sociodemográficas gênero, idade, escolaridade e renda. Além disso, associaram-se os escores obtidos por meio da Escala de Resiliência (Wagnild \& Young, 1993) com outros dois instrumentos: Percepção Subjetiva de Queixas de Memória e Mini Exame do Estado Mental (Folstei, Folstein \& McHugh, 1975).
Método

O enfoque metodológico utilizado neste estudo caracteriza-o como uma pesquisa quantitativa com delineamento transversal. Este foi um subprojeto de um projeto maior denominado "Idosos de Porto Alegre". Este estudo teve como objetivo principal avaliar aspectos biopsicossociais de uma amostra de idosos de ambos os sexos residentes na cidade de Porto Alegre, por intermédio de uma equipe interdisciplinar/transdisciplinar.

A amostra foi constituída por 86 idosos, 67 do sexo feminino e 19 do sexo masculino, com idade variando entre 60 e 90 anos. Os critérios de inclusão na amostra foram: ter entre 60 e 95 anos, ser residente da região metropolitana de Porto Alegre e participante do projeto de idosos de Porto Alegre. A Organização Mundial da Saúde (OMS, 1984) recomenda que seja utilizada a idade a partir dos 60 anos para considerar a fase da velhice em países em desenvolvimento como o Brasil. Esta é uma idade que pode ser atingida por parte significativa da população.

Nesse sentido, foram utilizados os seguintes instrumentos nesta investigação: questionário com dados sociodemográficos, Escala de Resiliência, Miniexame do Estado Mental (MMSE), Percepção Subjetiva de Queixa de Memória (MAC-Q do idoso). A escolha destes instrumentos esteve relacionada ao fato de serem objetivos, precisos, concisos, de fácil utilização e, consequentemente, de fácil avaliação, apresentando, desta forma, bons resultados de validade e consistência na avaliação dos aspectos que foram considerados no estudo.

No Brasil, a Escala de Resiliência foi adaptada recentemente por Pesce, Assis, Avanci, Santos, Malaquias e Carvalhaes (2005). O estudo de adaptação transcultural do instrumento foi realizado com uma amostra de 997 alunos com idades entre 12 e 19 anos, de ambos os sexos, matriculados em escolas públicas do município de São Gonzalo (RJ). Deste total, 485 eram alunos de $7^{a}$ e $8^{a}$ séries do ensino fundamental e 512 eram do $1^{\circ}$ e $2^{\circ}$ ano do ensino médio. Para a etapa de teste-reteste, uma amostra não probabilística reduzida de 203 alunos da amostra total (n=997) respondeu ao instrumento. A adaptação realizou-se com base nos seguintes critérios para a aceitação de equivalência entre instrumentos: conceitual, de itens, semântica, opera- 
cional, de mensuração (consistência interna expressa pelo alpha de Cronbach, coeficiente de correlação intraclasse para variáveis contínuas e estatística kappa ponderado para variáveis de escala ordinal); além da validade de conteúdo, de construto, análise fatorial e equivalência funcional (Pesce et al., 2005).

Em 1992, Crook, Feher e Larrabee propuseram o Questionário de Medida de Queixa Subjetiva de Memória como um instrumento autoadministrado; este instrumento foi validado por Bertolucci, em 1994. Ele é constituído por seis perguntas que visam a avaliar queixas de memória relacionadas a cinco situações do cotidiano - por exemplo: lembrar números de telefones ou códigos que você usa diária ou semanalmente -, com uma pergunta direcionada ao desempenho mnêmico global. É solicitado que seja feita uma comparação entre o desempenho mnêmico atual e aquele vivenciado aos 40 anos de idade. As respostas variam de "muito pior agora"a a"muito melhor agora", com cinco diferentes possibilidades de respostas. Uma pontuação igual ou superior a 25 pontos é considerada indicativa de disfunção subjetiva de memória.

No Brasil, o MMSE foi traduzido por Bertolucci et al. (1994), que observaram que o escore total do MMSE dependia do nível educacional dos 530 indivíduos avaliados na triagem médica de um hospital-escola de São Paulo. Eles propuseram a utilização de pontos de cortes diferenciados de acordo com a escolaridade para o diagnóstico genérico de "declínio cognitivo". O MMSE, desenvolvido por Folstein et al. (1975), é composto por questões agrupadas em sete categorias, planejadas para avaliar funções cognitivas específicas: orientação para tempo (5 pontos), orientação para local (5 pontos), registro de três palavras (3 pontos), atenção e cálculo (5 pontos), lembrança das três palavras (3 pontos), linguagem (8 pontos) e capacidade construtiva visual (1 ponto). 0 escore do MMSE pode variar de um mínimo de zero até um total máximo de 30 pontos.

Os instrumentos de coleta de dados foram aplicados pela própria pesquisadora, individualmente em cada idoso, em um ambulatório, em sala apropriada, ou em sua residência, quando os participantes estavam impossibilitados de ir até o ambulatório. Inicialmente foi preenchida a Ficha Sociodemográfica e o Miniexame do Estado Mental (MMSE). Os participantes que atenderam aos critérios de inclusão e não apresentaram prejuízo cognitivo foram submetidos ao teste de Percepção Subjetiva de Queixa de Memória e Escala de Resiliência.

Os dados quantitativos foram descritos por média e desvio-padrão. As variáveis categóricas foram apresentadas através de contagens e percentuais. A comparação do escore de resiliência foi realizada com teste $t$ de Student no caso de dois grupos. Para comparar médias de três ou mais grupos, foi utilizada a Análise de Variância (ANOVA) One-Way. O nível de significância adotado foi de $\alpha=0,05$.

Esta pesquisa foi aprovado pelo Comitê de Ética da Pontifícia Universidade Católica do Rio Grande do Sul (protocolo n 06/03409).

\section{Resultados}

Os resultados das variáveis sociodemográficas permitiram identificar que, dos 86 idosos entrevistados, $67(77,9 \%)$ eram do sexo feminino e 19 (22,1\%) do masculino. As idades variaram de 60 a 90 anos, sendo que a média de idade ficou em 70,4, com um desvio-padrão de 6,96 .

O estado civil predominante foi de casados (52 idosos), 11 eram solteiros, 22 eram viúvos e apenas um era separado. Em relação à origem, a brasileira foi maioria (48 idosos), seguida da italiana (22 idosos). Em termos de escolaridade, o estudo revelou um dado importante: mais de $70 \%$ possuíam menos de oito anos de estudo, e somente $23,3 \%$ tinham mais de oito anos de escolaridade. Dos idosos, 96,5\% relataram escrever com a mão direita e todos sabiam ler.

Quanto ao fator familiar (relacionado a quais pessoas moravam com o idoso), 35 (40,7\%) deles residiam com o cônjuge e filho; 18 (20,9\%) moravam somente com o cônjuge; 13 (15,1\%) moravam com outros parentes (sobrinhos, netos, irmãos); 12 (14,0\%) moravam sozinhos; e $8(9,3 \%)$ residiam com filhos. A maioria dos idosos relatou possuir residência própria (89,5\%), sendo $58(67,4 \%)$ residentes há mais de 20 anos na mesma moradia.

Quando solicitados a avaliar a saúde, 46 (53,5\%) idosos responderam"regular"; 38 (44,2\%) responderam "boa"; e 2 (2,3\%) avaliaram-na como "ruim". Quando questionados se, de um mês para cá, haviam deixado 
de realizar alguma atividade por motivos de saúde, 51 (59,3\%) responderam que "não" e 35 (40,7\%) responderam que "sim". A maioria dos entrevistados (59,3\%) possuía 2 a 3 hospitalizações prévias no último ano de vida.

No item hábito de fumar, foi observado que 65 $(75,6 \%)$ nunca fumaram e 21 pessoas $(24,4 \%)$ eram fumantes. Quanto à bebida alcoólica, 66 (76,7\%) não bebiam e 20 (23,3\%) afirmaram beber regularmente.

Em relação à preferência por alguma atividade em seus momentos de lazer, a maior preferência por parte das mulheres idosas foi de atividades manuais, igreja e novela. Já os homens idosos preferiram caminhada, leitura, igreja e novela, sendo que as duas primeiras foram as atividades em que eles se destacaram em relação às mulheres.

No que diz respeito à última renda mensal, os dados ficaram assim distribuídos: 46 (53,5\%) recebiam de um a dois salários-mínimos; 27 (31,4\%) recebiam de três a quatro salários-mínimos; sete $(8,1 \%)$ recebiam de cinco a seis salários-mínimos; e seis (7\%) recebiam sete ou mais salários-mínimos. Mais da metade, 65 pessoas (75,6\%), relatou que a principal origem de renda era a aposentadoria. A principal despesa que os idosos relataram ter foi com a habitação: 39 pessoas (45,3\%). Em relação à escala de resiliência, observou-se que a pontuação mínima, na escala normatizada de 0 a 100, foi 55,33; e a máxima, 96,67. Ao investigar a resiliência dos idosos, foi obtida a Média (M) de 84,9, Desvio-Padrão (DP) de 7,2. Cabe lembrar que os escores mais altos indicam nível de resiliência maior (Tabela 1).

Quanto à variável idade, 47 idosos pertenciam à faixa etária de 60 a 69 anos e apresentaram um escore médio de resiliência de 85,4, (DP=6,17) 27 idosos possuíam idade entre 70 e 79, apresentando média de resiliência 84,3, (DP=8,32); e 12 idosos pertenciam à faixa etária dos 80 anos ou mais, apresentando média de resiliência de 85,1, (DP=8,27).

A pontuação máxima na escala de resiliência dos idosos entre 60 e 69 anos foi de 96,0, e a mínima, de 67,3; nos idosos pertencentes à faixa etária de 70 a 79 anos, a pontuação máxima foi de 96,67, e a mínima, de 55,33; já nos idosos com 80 anos ou mais, a pontuação máxima atingiu 94,67, e a mínima, 65,33. A partir dos resultados observou-se que em idosos não houve alteração importante no valor da resiliência segundo a idade.

A correlação entre resiliência e sexo mostrou que a resiliência nos idosos não esteve relacionada significativamente ao sexo. Constatou-se que 67 idosos do sexo feminino apresentaram média de resiliência de 85,2 , (DP=7,52), enquanto os do sexo masculino apresentaram uma média de 84,2, (DP=5,7).

Tabela 1. Comparação entre as variáveis sociodemográficas e a escala de resiliência. Porto Alegre (RS), 2007.

\begin{tabular}{|c|c|c|c|c|}
\hline \multirow{2}{*}{ Variáveis } & \multicolumn{3}{|c|}{ Escala de Resiliência padronizada (0-100) } & \multirow{2}{*}{$p$} \\
\hline & $n$ & Média & Desvio-padrão & \\
\hline \multicolumn{5}{|l|}{ Faixa etária (anos) } \\
\hline $60-69$ & 47 & 85,4 & 6,17 & $0,796^{*}$ \\
\hline $70-79$ & 27 & 84,3 & 8,32 & \\
\hline$\geq 80$ & 12 & 85,1 & 8,27 & \\
\hline \multicolumn{5}{|l|}{ Sexo } \\
\hline Masculino & 19 & 84,2 & 5,70 & $0,593^{* *}$ \\
\hline Feminino & 67 & 85,2 & 7,52 & \\
\hline \multicolumn{5}{|l|}{ Escolaridade (anos) } \\
\hline 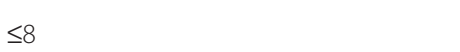 & 66 & 85,2 & 6,73 & $0,624^{* *}$ \\
\hline$>8$ & 20 & 84,3 & 8,48 & \\
\hline Última renda mensal (salário-mínimo) & & & 7,52 & $0,954^{* *}$ \\
\hline $1-4$ & 73 & 85,0 & 4,64 & \\
\hline$\geq 5$ & 13 & 85,1 & & \\
\hline
\end{tabular}


Dos 66 idosos possuíam escolaridade até o fundamental, sendo a média de resiliência 85,2, (DP=6,73); e 20 vinte idosos possuíam segundo grau ou superior, com média de resiliência de 84,3 (DP=8,5) (Tabela 1).

No que tange à renda econômica mensal dos idosos e sua relação com a escala de resiliência, os seguintes dados foram obtidos: dos 73 idosos que ganhavam de um a quatro salários-mínimos, a média de resiliência foi de 85,0 (DP=7,52), e dos 13 idosos que ganhavam cinco ou mais salários-mínimos, a média foi de 85,1 (DP=4,64) (Tabela 1).

Correlacionando o resultado obtido na Escala de Resiliência com os resultados obtidos na Percepção Subjetiva de Queixas de Memória, constatou-se que um número menos elevado de queixas de problemas de memória correspondeu a altos índices de resiliência, à medida que houve uma correlação inversa $(r=-0,270$; $p=0,012$ ).

Os resultados evidenciaram uma correlação direta entre a Escala de Resiliência e o desempenho no MEEM. Assim, os idosos que apresentaram melhor desempenho no MEEM mostraram-se mais resilientes $(r=0,281 ; p=0,009)$.

\section{Discussão}

Os idosos pesquisados eram, na maioria, do sexo feminino, o que comprova o fenômeno de feminização do processo do envelhecimento. De acordo com Netto (2002), nas sociedades pacíficas, a quantidade de homens e mulheres é quase igual até os 45 anos; a partir daí, os homens morrem em um ritmo mais rápido, de modo que, aos 70 anos, há aproximadamente seis mulheres para cada cinco homens e, aos 80 anos, esta razão é de 4:1.

Segundo dados do Instituto Brasileiro de Geografia e Estatística (2003), em 1991, as mulheres correspondiam a 54,0\% da população de idosos, passando para 55,1\% em 2000. Isto significa que para cada cem mulheres idosas havia 81,6 homens idosos, relação que, em 1991, era de 100 para 85,2. Elas vivem, em média, oito anos a mais que os homens.

Há muitas razões para a morte precoce dos 460 homens. Uma concepção popular atribui esta morte à vida fisicamente mais cansativa que os homens tradicionalmente levam. Mas uma complexa interação de fatores físicos, ambientais e diferenças no comportamento social também podem desempenhar um papel importante (Lang, Arnold \& Kupfer, 1994). Entretanto, há outras visões mais otimistas em relação à condição atual das mulheres idosas. Debert (1999), por exemplo, acredita que, para as idosas, tanto a velhice quanto a viuvez podem representar certa independência ou mesmo uma forma de realização.

Outro aspecto que chamou a atenção foi a baixa escolaridade encontrada nos idosos participantes desta pesquisa. Esse dado também está presente no estudo realizado pelo Instituto Brasileiro de Geografia e Estatística (2003), que relata que, nas décadas de 1930 até, pelo menos, os anos 1950, o ensino fundamental ainda era restrito a segmentos sociais específicos. Nessa medida, o baixo saldo da escolaridade média dessa população é um reflexo desse acesso desigual. Mas esse fenômeno vem mudando nos últimos anos. A proporção de idosos alfabetizados passou de 55,8\%, em 1991, para $64,8 \%$, em 2000, representando um crescimento de $16,1 \%$ no período.

A maioria dos idosos entrevistados possuía duas a três hospitalizações prévias no último ano. Este fato também foi observado no estudo de Lima-Costa, Barreto e Giatti (2003) sobre as condições de saúde e a capacidade funcional da população idosa brasileira. Segundo a Pesquisa Nacional por Amostra de Domicílio (PNDA) de 1998,69\% dos participantes relataram ter pelo menos uma doença crônica (sendo esta proporção maior entre as mulheres). Por outro lado, a presença destas doenças, a prevalência de incapacidade funcional e a ocorrência de internações aumentaram com a idade.

A maior preferência dos idosos em seus momentos de lazer recaiu em assistir programas de televisão (novela), seguidos de atividades religiosas e manuais. Este fato talvez se deva à falta de espaços de lazer específicos para esta população. Mazo, Lopes e Benedetti (2001) chamam a atenção dos órgãos governamentais para o estabelecimento de áreas físicas e atividades de lazer direcionadas aos idosos, em cumprimento à Política Nacional do Idoso. Também são relevantes as questões de acessibilidade: presença de rampas, corrimões, piso antiderrapante, e outras, nessas áreas físicas. 
Em relação à renda do idoso, verificou-se que a maioria era de aposentados e que mais da metade possuía uma renda mensal de um a dois salários-mínimos. Mesmo considerando que são muito baixos os valores das aposentadorias e pensões dos idosos brasileiros, estas muitas vezes constituem a principal fonte de renda, ou a única fonte de renda de toda a família sob responsabilidade do idoso.

Quanto à população brasileira, no que se refere ao fator econômico, desenvolveu-se um estudo (Barreto, Giatti, Lima-Costa \& Uchoa, 2003) sobre a influência da situação socioeconômica na saúde dos idosos que também se mostrou controverso. Foram utilizados dados da Pesquisa Nacional por Amostra de Domicílios de 1998 para examinar essa influência em uma amostra de 19068 idosos representativos da população brasileira com mais de 65 anos de idade. Os idosos com renda mais baixa apresentaram piores condições de saúde (pior percepção da saúde, interrupção de atividades por problemas de saúde, ter estado acamado e relato de algumas doenças crônicas), pior função física (avaliada por meio de seis indicadores) e menor uso de serviços de saúde (menor procura e menos visitas a médicos e dentistas).

Tais resultados não confirmaram observações realizadas em alguns países desenvolvidos quanto à ausência de associação entre o nível socioeconômico e a saúde do idoso. Ao contrário, os resultados desse estudo mostraram que mesmo pequenas diferenças na renda domiciliar seriam suficientemente sensíveis para identificar idosos com piores condições de saúde e menor acesso aos serviços de saúde no Brasil.

Quanto à resiliência dos idosos, observou-se que a média obtida por meio da Escala de Resiliência foi alta $(84,9)$; isto mostra que os idosos pesquisados conseguiram superar as adversidades que surgiram em suas vidas até o momento, pois a resiliência é uma maneira de sobreviver relacionada à integridade, adaptabilidade e tenacidade.

Este estudo mostrou que a correlação entre resiliência e idade não foi significativa. Em contrapartida, Lundman (2007) realizou uma pesquisa na qual o objetivo era investigar resiliência e sua relação com idade e gênero, utilizando também a escala de resiliência. A análise foi baseada em 1719 participantes suecos com idade entre 19 e 103 anos. Os resultados apontaram que a resiliência esteve relacionada com idade, sendo que os mais velhos apresentaram altos índices de resiliência.

No que se refere ao gênero e sua relação com a resiliência, observou-se que as mulheres apresentaram uma média um pouco maior na escala, quando comparado com os homens, mas não foi uma diferença importante, o que corroborou o estudo de Lundman (2007). Esse autor realizou seu estudo com 1719 participantes, 1248 mulheres e 471 homens, e constatou também que não houve relação entre gênero e resiliência.

A variável escolaridade, quando associada à escala de resiliência, não apresentou resultados importantes. Talvez isso tenha ocorrido em função de apenas 23,2 dos idosos possuírem maior escolaridade, o que representa dizer que ser resiliente independe de possuir ensino superior ou somente o ensino fundamental.

Em relação à renda econômica mensal dos idosos e sua relação com a resiliência, a média obtida por meio da Escala de Resiliência entre os idosos que ganhavam de um a cinco salários-mínimos e os que ganhavam cinco ou mais oscilou entre 84,95 e 84,46, ou seja, praticamente a mesma. Com este resultado percebe-se que a resiliência pode ser uma característica dos idosos pertencentes tanto à classe econômica social mais baixa quanto àquela mais alta. Não há muitos estudos relacionando resiliência e classe econômica, mas já existem estudos investigando esta relação.

Segundo pesquisa de Valdes et al. (2007), a classe social determinaria o ritmo de envelhecimento, fator que se sobrepõe a outros, como saúde, dieta e maus hábitos. Após analisar os casos de mais de 1500 mulheres gêmeas, cientistas chegaram à conclusão de que existiu uma relação entre classe social e o nível de deterioração celular no corpo. O stress poderia ser a chave para explicar a aceleração do envelhecimento. As pessoas de classes sociais mais baixas tiveram maior probabilidade de se sentir pouco seguras (principalmente no trabalho), de ter autoestima baixa e de sentir que não controlavam a própria vida, aspectos que causariam um stress que danificaria as células e aceleraria o ritmo de envelhecimento.

Na correlação entre Escala de Resiliência e Percepção Subjetiva de Queixas de Memória, foi encontrada associação significativa e inversa. À medida que o 
número de queixas de problemas de memória era menos elevado, mais altos eram os índices obtidos na Escala de Resiliência.

O estudo apontou, também, uma correlação direta e significativa entre a Escala de Resiliência e o Miniexame do Estado Mental. Assim, quanto mais altos os escores de resiliência, maior foi o desempenho cognitivo (orientação para tempo e espaço, memória, linguagem etc.) entre os idosos.

Portanto, a partir dos dados apresentados percebe-se, entre outros aspectos, que a resiliência independe de algumas variáveis, o que leva a pensar que a resiliência não depende de traços e disposições pessoais apriorísticos, nem se manifesta apenas a partir da superação de fatores de risco predeterminados (Dell'Aglio, Koller \& Yunes, 2006). Masten (2001) argumentou que resiliência seria um processo normativo da adaptação, presente na espécie humana e aplicável ao desenvolvimento em ambientes favoráveis ou adversos. Desta forma, pertence a todos os seres vivos essa "capacidade" para o desenvolvimento saudável e positivo.

\section{Considerações Finais}

Este trabalho propôs-se a estudar a resiliência em idosos e sua relação com algumas variáveis sociodemográficas como gênero, idade, escolaridade e renda. Dispôs-se também a avaliar a relação entre resiliência e desempenho cognitivo.

Por não haver um ponto de corte na Escala de Resiliência, o seu escore foi normatizado de 0 a 100. Com esta normatização, observou-se uma média alta de resiliência nesta população de idosos.

O estudo revelou que a resiliência não foi associada às variáveis sociodemográficas gênero, idade, escolaridade e renda.

Correlacionando o resultado obtido na Escala de Resiliência com resultados obtidos na Percepção Subjetiva de Queixas de Memória, constatou-se que um número mais elevado de queixas de problemas de memória correspondeu a baixos índices de resiliência, à medida que houve uma correlação inversa e signifi-

462 cativa entre estes resultados.
Assim, houve uma correlação direta e significativa entre a Escala de Resiliência e o Miniexame do Estado Mental, isto é, quanto mais resiliente o idoso se apresentou, melhor encontrou-se a sua memória.

A limitação deste estudo foi o fato de ser transversal, representando um recorte da amostra estudada, e também acabou não sendo possível saber qual fator foi determinante para a ocorrência do outro: se houve um melhor desempenho cognitivo devido à alta resiliência que o idoso possuía ou vice-versa.

Apesar da ampla discussão em outros países, no Brasil o termo resiliência ainda não foi bem incorporado pelo meio acadêmico, visto o pequeno número de publicações sobre o tema. Nesse sentido, é de suma importância que outros trabalhos possam ser desenvolvidos nesta área.

É importante salientar a necessidade de implantações de programas que visem à manutenção das capacidades cognitivas e funcionais dos idosos, proporcionando, assim, uma melhoria na autoestima e, consequentemente, na qualidade de vida do idoso. Tais programas devem apresentar caráter multidisciplinar e se fundamentar na implantação de alternativas de participação, ocupação e convívio do idoso com as demais gerações.

Diante do exposto, espera-se que este estudo possa ter reunido informações que contribuam para ampliar os atuais níveis de conhecimento na área sobre resiliência no envelhecimento humano, e que os resultados possam servir como referencial para futuras pesquisas, auxiliando, desta forma, os profissionais da saúde e a população, de um modo geral, no que se refere aos cuidados com idosos.

\section{Referências}

Barreto, S., Giatti, L., Lima-Costa, M. F., \& Uchoa, E. (2003). Desigualdade social e saúde entre idosos brasileiros: um estudo na Pesquisa Nacional por Amostra de Domicílios. Cadernos de Saúde Pública, 19 (3), 745-757.

Bertolucci, P. H. F., Brucki, S. M. D., Campacci, S. R., \& Juliano, Y. (1994). O Mini-exame do estado mental em uma população geral: impacto da escolaridade. Arquivos de Neuropsiquiatria, 5, 1-7.

Costa, M. F. F. L., Guerra, H. L., Barreto, S. M., \& Guimarães, R. M. (2000). Diagnóstico da situação de saúde da população idosa brasileira: um estudo da mortalidade e das inter- 
nações hospitalares públicas. Informe Epidemiológico do SUS, 9 (1): 23-41.

Debert, G. G. (1999). A reinvenção da velhice. São Paulo: EdUSP.

Dell'Aglio, D. D., Koller, S. H., \& Yunes, M. A. (2006). Resiliência epsicologia positiva: interfaces do risco à proteção. São Paulo: Casa do Psicólogo.

Ferreira, A. B. H. (1975). Novo dicionário da língua portuguesa. Rio de Janeiro: Nova Fronteira.

Folstein, M. F., Folstein, S. E., \& McHugh, P. R. (1975). Mini- Mental State: a pratical method for grading the cognitive state of patients for the clinician. Journal of Psychiatric Research, 12, 189-198.

Giuliani, P. C. (1997). Um estudo analítico sobre a evolução do perfil do executivo. Dissertação de mestrado não-publicada, Pontifícia Universidade Católica de São Paulo.

Hoppe, M. M.W. (1998). Redes de apoio social e afetivo de crianças expostas a situações de risco. Dissertação de mestrado não-publicada, Programa de Pós-Graduação em Psicologia, Universidade Federal do Rio Grande do Sul, Porto Alegre.

Hutz, C. S., \& Koller, S. H. (1996). Questões sobre o desenvolvimento de crianças em situação de rua. Estudos de Psicologia (Natal), 2 (1), 175-197.

Hutz, C. S., Koller, S. H., \& Bandeira, D. R. (1996). Resiliência e vulnerabilidade em crianças em situação de risco. Coletâneas da ANPEPP, 1 (12), 79-86.

Instituto Brasileiro de Geografia e Estatística. (2003). Pesquisa nacional por amostra de domicílios 2002: microdados. Rio de Janeiro: IBGE.

Lang, E., Arnold, K., \& Kupfer, P. (1994). Women live longer biological, medical and socilogic causes. Zeitzchrift fuer gerontologic, 27, 10-15.

Lima-Costa, M. F., Barreto, S. M., \& Giatti, L. (2003). Condições de saúde, capacidade funcional, uso de serviços de saúde e gastos com medicamentos da população idosa brasileira: um estudo descritivo baseado na Pesquisa Nacional por Amostra de Domicílios. Cadernos de Saúde Pública, 19 (3), 735-743.

Lindström, B. (2001). O significado de resiliência. Adolescência Latino-Americana, 2, 133-137.

Lundman, B. (2007). Psychometric properties of the swedish version of the resilience scale. Scandinavian Journal of Caring Science, 21 (2), 229-237.

Masten, A. (2001). Ordinary magic: resilience processes in development. American Psychologist, 56 (3), 227-238.

Mazo, G. Z., Lopoes, M. A., \& Benedetti, T. B. (2001). Atividade física e o idoso. Porto Alegre: Sulina.

Melillo, A., Suárez-Ojeda, E. N., \& Rodríguez, D. (Orgs.) (2004). Resiliencia y subjetividad: Ios ciclos de la vida. Buenos Aires: Paidos.
Neri, A. L. (2001). Velhice e qualidade de vida na mulher. In A. L. Neri (Org.), Desenvolvimento e envelhecimento: perspectivas biológicas, psicológicas e sociológicas (pp.161-200). Campinas: Papirus.

Netto, M. P. (2002). O estudo da velhice no século XX: histórico, definição do campo e termos básicos. In E. V. Freitas, P. Y. Ligia, F. A. X. Cançado \& M. L. Gorzoni. Tratado de geriatria e gerontologia Rio de Janeiro (pp.2-12) Rio de Janeiro: Guanabara Koogan.

Organización Mundial de la Salud. (1984). Aplicaciones de la epidemiologia al estudio de los ancianos: informe de un grupo cientifico de la OMS sobre a epidemiologia del envejecimento. (Série de Informes Técnicos, 706). Genebra: OMS.

Pesce, R., Assis, S. G., Avanci, J., Malaquias, J., \& Oliveira, R. V. C. (2005). Adaptação transcultural, confiabilidade e validade da escala de resiliência. Cadernos de Saúde Pública, 21 (2), 436-448.

Pinheiro, D. P. N. (2004). A resiliência em discussão. Psicologia em Estudo, 9, 67-75.

Schaie, K. W., \& Hofer S. M. (2001). Longitudinal studies in research on aging. In J. E. Birren \& K. W. Schaie (Eds.), Handbook of the psychology of aging (pp.53-77). San Diego, CA: Academic Press.

Smith, J., Borchelt, M., Maier, H., \& Jopp, D. (2002). Health and well-being in the young and oldest old. Journal of Social Issues, 58 (4), 715-732.

Souza, M.T.S., \& Cerveny, C. M. O. (2006). Resiliência psicológica: revisão da literatura e análise da produção científica. Revista Interamericana de Psicología, 40 (1), 115-122.

Staudinger, U. M., Marsiske, M., \& Baltes, P. B. (1995). Resiliência e níveis de capacidade de reserva na velhice: perspectivas da teoria de curso de vida. In A. L. Neri (Org.), Psicologia do envelhecimento: temas relacionados na perspectiva de curso de vida (pp.195-228). Campinas: Papirus.

Valdes, A. M., Loughlin, J., VanOene, M., Chapman, K., Surdulescu, G. L., Doherty, M., et al. (2007). Sex and ethnic differences in the association of ASPN, CALM1, COL2A1, COMP and FRZB with genetic susceptibility to osteoarthritis of the knee. Arthritis \& Rheumatism, 56 (1), 137-146.

Vanistendael, S. (1999). Prévenir le trauma d'une agression sexuelle: un éclairage à partir de la résilience. In M. P. Poilpot (Org.), Souffrir et se construire (pp.107-123). Ramonville: Éditions Érès.

Wagnild, G. M., \& Young, H. M. (1993). Development and psychometric evaluation of resilience scale. Journal of Nursing Measurement, 1 (2), 165-178.

Recebido em: 7/11/2007

Versão final reapresentada em: 2/4/2008

Aprovado em: 5/3/2009 